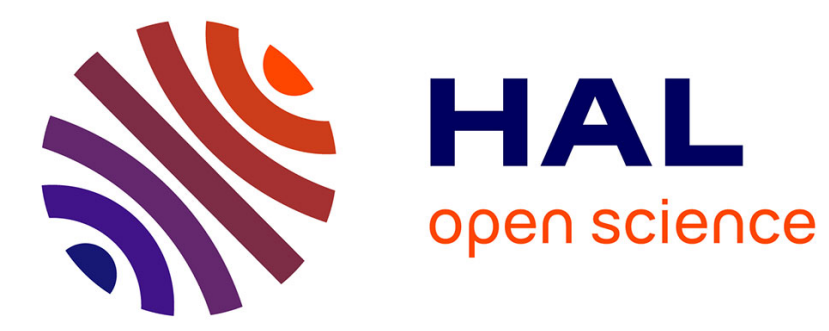

\title{
Quadratic response functions in the relativistic four-component Kohn-Sham approximation.
}

\author{
Johan Henriksson, Trond Saue, Patrick Norman
}

\section{To cite this version:}

Johan Henriksson, Trond Saue, Patrick Norman. Quadratic response functions in the relativistic fourcomponent Kohn-Sham approximation.. Journal of Chemical Physics, 2008, 128 (2), pp.024105 (1-9). 10.1063/1.2816709 . hal-00761414

\section{HAL Id: hal-00761414 \\ https://hal.science/hal-00761414}

Submitted on 21 Jan 2020

HAL is a multi-disciplinary open access archive for the deposit and dissemination of scientific research documents, whether they are published or not. The documents may come from teaching and research institutions in France or abroad, or from public or private research centers.
L'archive ouverte pluridisciplinaire HAL, est destinée au dépôt et à la diffusion de documents scientifiques de niveau recherche, publiés ou non, émanant des établissements d'enseignement et de recherche français ou étrangers, des laboratoires publics ou privés. 


\section{Quadratic response functions in the relativistic four-component Kohn-Sham approximation}

Cite as: J. Chem. Phys. 128, 024105 (2008); https://doi.org/10.1063/1.2816709

Submitted: 05 October 2007 . Accepted: 31 October 2007. Published Online: 10 January 2008

Johan Henriksson, Trond Saue, and Patrick Norman

ARTICLES YOU MAY BE INTERESTED IN

Density-functional theory of linear and nonlinear time-dependent molecular properties

The Journal of Chemical Physics 117, 9630 (2002); https://doi.org/10.1063/1.1516805

Optical nonlinearities in conjugated systems: $\beta$-carotene

Applied Physics Letters 23, 178 (1973); https://doi.org/10.1063/1.1654850

Role of noncollinear magnetization for the first-order electric-dipole hyperpolarizability at the four-component Kohn-Sham density functional theory level

The Journal of Chemical Physics 130, 024109 (2009); https://doi.org/10.1063/1.3054302

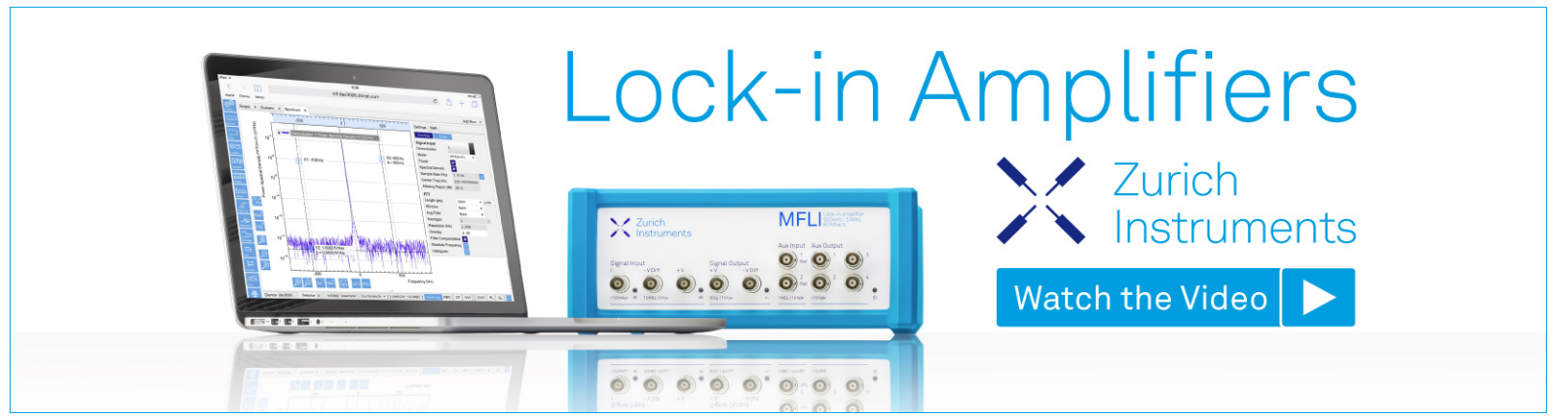

J. Chem. Phys. 128, 024105 (2008); https://doi.org/10.1063/1.2816709

(c) 2008 American Institute of Physics. 


\title{
Quadratic response functions in the relativistic four-component Kohn-Sham approximation
}

\author{
Johan Henriksson \\ Department of Physics, Chemistry and Biology, Linköping University, SE-581 83 Linköping, Sweden \\ Trond Saue \\ Institut de Chimie, UMR 7177 CNRS et Université Louis Pasteur, 4 rue Blaise Pascal, \\ F-67000 Strasbourg, France \\ Patrick Norman ${ }^{\text {a) }}$ \\ Department of Physics, Chemistry and Biology, Linköping University, SE-581 83 Linköping, Sweden
}

(Received 5 October 2007; accepted 31 October 2007; published online 10 January 2008)

\begin{abstract}
A formulation and implementation of the quadratic response function in the adiabatic four-component Kohn-Sham approximation is presented. The noninteracting reference state is time-reversal symmetric and formed from Kramers pair spinors, and the energy density is gradient corrected. Example calculations are presented for the optical properties of disubstituted halobenzenes in their meta and ortho conformations. It is demonstrated that correlation and relativistic effects are not additive, and it is shown that relativity alone reduces the $\mu \bar{\beta}$-response signal by $62 \%$ and $75 \%$ for meta- and ortho-bromobenzene, respectively, and enhances the same response by $17 \%$ and $21 \%$ for meta- and ortho-iodobenzene, respectively. Of the employed functionals, CAM-B3LYP shows the best performance and gives hyperpolarizabilities $\beta$ distinctly different from B3LYP. (C) 2008 American Institute of Physics. [DOI: 10.1063/1.2816709]
\end{abstract}

\section{INTRODUCTION}

In the presence of external (or internal) perturbing electromagnetic fields, the molecular polarization (or magnetization) can be expressed as a Taylor series in terms of the field strengths and the coupling parameters relate to spectroscopic properties (see, for instance, the book by Boyd). ${ }^{1}$ Over the past 20 years, increasingly accurate and efficient computational methods have been developed to determine the linear as well as nonlinear molecular response parameters and, today, theoretical calculations are routinely used for the interpretation of experimental spectra as well as for material functionalization and optimization. If we are concerned with the correction to the molecular polarization that depends quadratically on the perturbing electric-field strengths, we note fundamental nonlinear optical processes, such as the second-harmonic generation and the electro-optical Pockels effect, and an important device such as the optical parametric oscillator. Moreover, static magnetic fields can be used to induce birefringences in nonchiral systems and, in this context, the quadratic response parameters (or quadratic response functions) are pertinent to spectroscopies such as magnetic circular dichroism and the Faraday effect.

In nonrelativistic quantum chemistry, quadratic response functions have been formulated and implemented at the electron uncorrelated level in the so-called time-dependent Hartree-Fock (HF) approximation ${ }^{2-6}$ - this approximation is sometimes also referred to as the time-dependent coupled perturbed Hartree-Fock level or the random phase approximation - as well as at the electron correlated level

${ }^{a)}$ Electronic mail: panor@ifm.liu.se. employing second-order Møller-Plesset, ${ }^{7}$ multiconfiguration self-consistent field (MCSCF), ${ }^{2,6}$ and coupled cluster ${ }^{8-10}$ reference states. More recently, the quadratic response function has also been formulated in the second-order polarization propagator approach ${ }^{11}$ and formulated and implemented in Kohn-Sham density functional theory (DFT). ${ }^{12,13}$ Successful use of these computational techniques has been demonstrated in numerous publications in the literature and, furthermore, it has been shown that a residue analysis of the response functions at the electronic transition frequencies of the system enables the calculation of observables in absorption spectroscopies and properties of electronically excited states. ${ }^{2}$ At small frequency detunings of the perturbing fields, however, one must be cautious since the dispersion of the response functions is severely overestimated in this region due to the divergences at resonances. This issue has been considered in a series of publications by Norman et al., and a resonance convergent formulation of response theory up to second order has been proposed and implemented at the HF, MCSCF, and DFT levels of theory. ${ }^{14}$ It stands clear that, in the nonrelativistic realm, there exist sophisticated and highly accurate methodologies and program implementations for the determination of the linear and nonlinear responses in the electronic density to time-dependent electromagnetic field perturbations.

In photonics, it is well-known that the use of organometallic compounds can give unprecedented performance in certain applications, ${ }^{15}$ but it is clear that, from a theoretical perspective, the inclusion of one or several heavy atoms calls for the treatment of relativistic effects in one way or another. It is possible to consider these effects by perturbation theory and, in the nonrelativistic framework, to add relativistic cor- 
rections to the perturbation operator that describes the coupling to the external electromagnetic fields. This approach has been adopted to determine, e.g., phosphorescence, ${ }^{16,17}$ electron spin resonance, ${ }^{18}$ and nuclear magnetic resonance parameters $^{19}$ but, although these calculations employ quadratic response functions, they are all examples of secondorder molecular properties since one of the perturbation operators in the response functions refers to an intrinsic field. In order to address a third-order molecular property in a relativistic-perturbational approach, one would need to evaluate a cubic response function which is computationally more complex, and we are not aware of such work. Apart from the increased computational complexity, a relativisticperturbational approach is also limited by the fact that it is not applicable when relativistic effects are large and, therefore, must be included in the zeroth-order Hamiltonian.

There exist a number of ways to include relativistic effects in the zeroth-order molecular Hamiltonian. One can replace the core electron densities by effective potentials and include only the valence electrons in the parametrization of the wave function. ${ }^{20}$ The parameters of the effective core potentials (ECPs) may be optimized against accurate relativistic atomic densities and later used in regular nonrelativistic calculations. The ECP approach indirectly accounts for scalar relativistic as well as spin-orbit effects in the atomic cores, and it can be applied to all elements of the Periodic Table while providing a reasonable accuracy (see Refs. 21-23 for evaluations of this method for third-order molecular properties). The obvious limitation is the neglect of direct (rather than indirect via the core potential) relativistic effects in the valence electron density. The severity of this approximation varies strongly for different molecular properties; not only does it vary with respect to the order of the molecular property but it also varies substantially for different properties of the same order. The most striking example when spinorbit effects in the valence electron density are of prominent importance in the calculation of a quadratic response function is given by the two-photon absorption spectra (which relate to a first-order residue of the quadratic response function). It was demonstrated by Henriksson et al. ${ }^{24}$ that even for a light element such as neon, the inclusion of spin-orbit interactions is necessary to obtain a qualitatively correct twophoton absorption spectrum.

At the all-electron level of theory, spin-free scalar relativistic corrections may be added to the one-electron Hamiltonian and, with the neglect of the picture change in the perturbation operators, response properties can be determined without further modifications of the nonrelativistic code. In this way, the hyperpolarizabilities of a series of group IIb sulfides were determined ${ }^{25}$ in the spin-averaged Douglas-Kroll approximation, as introduced by Hess, ${ }^{26,27}$ and the same approach has later been benchmarked against four-component calculations and then showing significant discrepancies for the hyperpolarizabilities of iodine and tellurium hydrides. ${ }^{21}$ Full inclusion of scalar relativistic and spin-orbit effects in the calculation of third-order molecular properties was accomplished with the implementation of the quadratic response function (and its first- and second-order residues) in the time-dependent four-component Hartree-
Fock approximation. ${ }^{24,28,29}$ While accurate with respect to relativistic effects, it is expected that the applicability of this method is severely limited due to the large effects of electron correlation on nonlinear response properties and since it is inappropriate to treat relativity and electron correlation separately. In the present work, we therefore develop and implement the quadratic response function in the time-dependent four-component Kohn-Sham DFT approximation. Our work should be seen as an extension of the previous mentioned work in the HF approximation ${ }^{24,28,29}$ as well as the work on the linear response function in the DFT approximation. ${ }^{30}$

In Sec. II A, we give a brief review of the fourcomponent Kohn-Sham approximation and the derivation of the quadratic response function but, since these general aspects are largely covered in our previous work, ${ }^{29,30}$ we focus primarily on a presentation of the details of the implementation that are unique to the extension made here (see Sec. II B). In Sec. III, we illustrate our implementation with an example calculation of the first-order electric-dipole hyperpolarizability for dibromo- and di-iodo-substituted benzene using a set of standard density functionals. We emphasize that although the implementation is completely general, we here present results for nonoscillating external perturbations which require functionals of the charge density only. For dynamic properties, we would like to perform a detailed investigation of also adding the induced noncollinear magnetization as a functional variable, and this work is in progress.

\section{THEORY AND METHODOLOGY}

\section{A. Time-dependent four-component Kohn-Sham approximation}

In the time-dependent four-component Kohn-Sham DFT approximation, the noninteracting reference system is described by a determinant of spinors $\phi_{p}(\mathbf{r})$. The time-reversal symmetric reference state of the isolated system $\left|0_{s}\right\rangle$ is variationally optimized with the use of an electronic Hamiltonian for the interacting system in which the kinetic energy is given by the free-particle Dirac Hamiltonian and the electron-electron repulsion is approximated by the instantaneous Coulomb interaction. This approximate form of the two-electron part of the relativistic Hamiltonian is improved on by the inclusion of the Gaunt term, or the full Breit interaction, but the introduction of current-current interactions in DFT requires a general consideration before introduced here. $^{31}$ Time-reversal symmetry of $\left|0_{s}\right\rangle$ is enforced by the occupation of Kramers pairs of spinors (corresponding to the closed-shell state in a nonrelativistic theory) - a pair of spinors are related by the time-reversal operator,

$$
\Phi_{\bar{p}}(\mathbf{r})=\hat{\mathcal{K}} \phi_{p}(\mathbf{r}), \quad \hat{\mathcal{K}}=\left[\begin{array}{cc}
0_{2} & -\mathbf{I}_{2} \\
\mathbf{I}_{2} & 0_{2}
\end{array}\right] \hat{\mathcal{K}}_{0},
$$

where $\hat{\mathcal{K}}_{0}$ denotes the complex conjugation operator. The eigenvalues of the spinors are divided into two sets that are separated by circa twice the electron rest energy, and spinors corresponding to the upper and lower sets are sometimes referred to as electronic and positronic orbitals, respectively (although they all represent electron wave functions). The 
reference state includes only electronic orbitals and is optimized in a minmax sense. ${ }^{32}$

When subjected to an external time-dependent electromagnetic field, the reference state becomes time dependent in a way that is not described by a trivial phase factor but involves electronic transitions to virtual orbitals. We parametrize this time dependence in a nonredundant and unitary way as follows: ${ }^{2,31}$

$$
\left|\tilde{0}_{s}(t)\right\rangle=\exp [-\hat{\kappa}(t)]\left|0_{s}\right\rangle, \quad \hat{\kappa}(t)=\kappa_{a i} \hat{a}_{a}^{\dagger} \hat{a}_{i}-\kappa_{a i}^{*} \hat{a}_{i}^{\dagger} \hat{a}_{a} .
$$

Here and in the following, we have made use of the Einstein summation convention for repeated indices and $a, b, \ldots, i, j, \ldots$, and $p, q, \ldots$ are indices of virtual, occupied, and general molecular orbitals, respectively. In general, the summation over virtual orbitals in Eq. (2) includes the positronic orbitals and the corresponding electron transfer amplitudes are at times denoted by $\kappa_{a i}^{-}$(to be distinguished from rotations among electronic orbitals with amplitudes $\kappa_{a i}^{+}$). In calculations of electric-field induced valence properties, such as the electric-dipole hyperpolarizability, the effect of redressing of the electronic states due to the inclusion of the $\kappa_{a i}^{-}$parameters in the propagator is small and can be ignored with the benefit of memory savings. ${ }^{23}$

For weak, periodic external fields, we can use the quasienergy formalism to determine the time dependence of the $\kappa$ parameters ${ }^{33}$ - a technique which was also used for the derivation and implementation of the linear response function at this level of theory ${ }^{30}$ (see, however, Ref. 34 for a discussion about the validity of this approach). The relevant time-averaged Kohn-Sham quasienergy functional can be written as

$$
Q[\rho]=T_{s}[\rho]+V[\rho]+J[\rho]+Q_{\mathrm{xc}}[\rho]+S_{s}[\rho],
$$

where the time-dependent electron density is introduced as $\rho$ and depends implicitly on the $\kappa$ parameters. The response functions are defined as derivatives of the quasienergy with respect to the Fourier amplitudes of the external electromagnetic field, and the third-order response, or the quadratic response function, is given by

$$
\langle\langle\hat{A} ; \hat{B}, \hat{C}\rangle\rangle_{\omega_{B}, \omega_{C}}=\left.\frac{d^{3} Q}{d \varepsilon_{A}\left(\omega_{A}\right) d \varepsilon_{B}\left(\omega_{B}\right) d \varepsilon_{C}\left(\omega_{C}\right)}\right|_{\varepsilon=0} .
$$

In evaluating this derivative, we note that the sum of terms in Eq. (3) excluding $Q_{\mathrm{xc}}$ corresponds formally to Hartree-Fock theory without exchange interaction. We can, therefore, benefit from the implementation of the quadratic response function reported in Ref. 29 and use it with a mere turnoff of the exchange interaction (or partial turnoff for hybrid functionals) together with the addition of the contribution from $Q_{\mathrm{xc}}$. We will adopt the adiabatic approximation and employ the time-dependent exchange-correlation functional as a substitute for $Q_{\mathrm{xc}}$,

$$
Q_{\mathrm{xc}}[\rho] \rightarrow E_{\mathrm{xc}}[\rho]=\int e_{\mathrm{xc}}(\rho, \zeta) d \tau
$$

The energy density is here assumed to be a function of $\rho$ and the square norm of the electron density gradient $\zeta=\nabla \rho \cdot \nabla \rho$, and a time averaging is implied here as well. The time aver- aging will impose that the response function [Eq. (4)] is nonzero only when $\omega_{A}=-\left(\omega_{B}+\omega_{C}\right)$. In the next section, we will present the detailed expressions needed for the implementation of the part in Eq. (4) that is due to $E_{\mathrm{xc}}$.

\section{B. Implementation of the exchange-correlation contribution to the quadratic response function}

In the evaluation of the third-order derivative of $E_{\mathrm{xc}}$ with respect to the amplitudes of the external fields, we will use chain rule differentiation of the energy density $e(\rho, \zeta)$. We will view the electron density as dependent on the $\kappa$ parameters and determine the response of the latter to the external perturbation from the variational condition $\delta Q[\rho]=0$. Since the $2 n+1$ rule applies in the present case, it will be sufficient to determine the first-order response in $\kappa$ with respect to $\varepsilon(\omega)$ in order to determine the quadratic response function. We note that the details and code implementation concerned with the determination of this linear response have already been considered in Ref. 30. The structure of the implementation of the quadratic response function at the Hartree-Fock level is such that first, the formation of a generalized electronic gradient

$$
\left.\frac{\partial}{\partial \kappa_{a i}} \frac{d^{2} E_{\mathrm{xc}}}{d \varepsilon_{B} d \varepsilon_{C}}\right|_{\varepsilon=0}
$$

is made and, thereafter, this gradient is contracted with the response of the $\kappa_{a i}$ parameters with respect to the external field. ${ }^{29}$ In order to comply with this structure, we therefore seek an explicit expression for the quantity in Eq. (6).

This exchange-correlation contribution will be added to the generalized gradient as due to the Coulomb interaction and which is denoted as $E^{[3]} N^{B} N^{C}$ in Ref. 29. Let us now turn to the differentiation of $E_{\mathrm{xc}}$ and first consider the partial derivatives that will appear. In doing so, we will make use of the fact that $\varepsilon \rightarrow 0$ implies that $\kappa \rightarrow 0$ and vice versa and, although partial differentiation is to be made independently for $\kappa_{a i}$ and $\kappa_{a i}^{*}$, we restrict the presentation to include only one of them.

With the use of the density operator,

$$
\hat{\rho}=\Omega_{p q} \hat{a}_{p}^{\dagger} \hat{a}_{q}, \quad \Omega_{p q}=\phi_{p}^{\dagger}(\mathbf{r}) \phi_{q}(\mathbf{r}),
$$

the electron density can be written as

$$
\rho(\mathbf{r})=\left\langle\tilde{0}_{s}|\hat{\rho}| \tilde{0}_{s}\right\rangle=\Omega_{p q}\left\langle 0_{s}\left|e^{\hat{\kappa}} \hat{a}_{p}^{\dagger} \hat{a}_{q} e^{-\hat{\kappa}}\right| 0_{s}\right\rangle .
$$

Expanding the density matrix elements with use of the Baker-Campbell-Hausdorff expansion yields

$$
\rho=\sum_{n=0}^{\infty} \rho^{(n)}, \quad \rho^{(n)}=\frac{\Omega_{p q}}{n !}\left\langle 0_{s}\left|\hat{\boldsymbol{\kappa}}^{n} \hat{a}_{p}^{\dagger} \hat{a}_{q}\right| 0_{s}\right\rangle,
$$

where the action of the superoperator $\hat{\boldsymbol{\kappa}}$ is the formation of a commutator according to $\hat{\boldsymbol{\kappa}} \hat{A}=[\hat{\kappa}, \hat{A}]$. The differentiation of the density with respect to the external fields gives

$$
\rho^{A B C \cdots}:=\left.\frac{\partial^{n} \rho}{\partial \varepsilon_{A} \partial \varepsilon_{B} \partial \varepsilon_{C} \cdots}\right|_{\varepsilon=0}=\Omega_{a i}^{B C \cdots} \frac{\partial \kappa_{a i}}{\partial \varepsilon_{A}},
$$

where we have introduced 


$$
\Omega_{a i}^{B C \cdots}:=\frac{\partial^{n} \rho^{(n)}}{\partial \kappa_{a i} \partial \kappa_{b j} \partial \kappa_{c k} \cdots} \frac{\partial \kappa_{b j}}{\partial \varepsilon_{B}} \frac{\partial \kappa_{c k}}{\partial \varepsilon_{C}} \cdots
$$

In order to account for gradient-corrected density functionals, we also introduce the following partial derivatives of $\zeta$ with respect to the external field:

$$
\begin{aligned}
& \zeta^{B}:=\left.\frac{\partial \zeta}{\partial \varepsilon_{B}}\right|_{\varepsilon=0}=2 \nabla \rho \cdot \nabla \rho^{B}, \\
& \zeta^{B C}:=\left.\frac{\partial^{2} \zeta}{\partial \varepsilon_{B} \partial \varepsilon_{C}}\right|_{\varepsilon=0}=2\left(\nabla \rho \cdot \nabla \rho^{B C}+\nabla \rho^{B} \cdot \nabla \rho^{C}\right) .
\end{aligned}
$$

The numerical grid-integration kernel in the program assumes the integrand to be written on the form

$$
{ }_{s} \Omega_{p q}+\mathbf{v} \cdot \nabla \Omega_{p q},
$$

where the scalar $s$ and vectorial $\mathbf{v}$ functions as well as the atomic orbital density matrix corresponding to $\Omega_{p q}$ are to be specified for a given property integration. Let us illustrate how this works for the electronic gradient,

$$
\begin{aligned}
\left.\frac{\partial E_{\mathrm{xc}}}{\partial \kappa_{a i}^{*}}\right|_{\kappa=0} ^{*} & =\int\left[\left.\frac{\partial e_{\mathrm{xc}}}{\partial \rho} \frac{\partial \rho}{\partial \kappa_{a i}^{*}}\right|_{\kappa=0}+\left.\frac{\partial e_{\mathrm{xc}}}{\partial \zeta} \frac{\partial \zeta}{\partial \kappa_{a i}^{*}}\right|_{\kappa=0}\right] d \tau \\
& =-\int f_{\mathrm{xc} ; a i} d \tau
\end{aligned}
$$

in which appears the exchange-correlation part of the KohnSham matrix,

$$
\begin{aligned}
& f_{\mathrm{xc} ; p q}=s_{f} \Omega_{p q}+\mathbf{v}_{f} \cdot \nabla \Omega_{p q}, \\
& s_{f}=\frac{\partial e_{\mathrm{xc}}}{\partial \rho}, \quad \mathbf{v}_{f}=\frac{1}{|\nabla \rho|} \frac{\partial e_{\mathrm{xc}}}{\partial|\nabla \rho|} \nabla \rho .
\end{aligned}
$$

Continuing to linear response, we get

$$
\left.\frac{\partial}{\partial \kappa_{a i}^{*}} \frac{\partial E_{\mathrm{xc}}}{\partial \varepsilon_{B}}\right|_{\varepsilon=0}=-\int\left(f_{\mathrm{xc} ; a i}^{B}+g_{\mathrm{xc} ; a i}^{[B]}\right) d \tau,
$$

where the integrand consists of the exchange-correlation part of the one-index transformed Kohn-Sham matrix,

$$
f_{\mathrm{xc} ; p q}^{B}=s_{f} \Omega_{p q}^{B}+\mathbf{v}_{f} \cdot \nabla \Omega_{p q}^{B},
$$

and a remainder that equals to

$$
\begin{aligned}
g_{\mathrm{xc} ; p q}^{[B]} & =s_{g} \Omega_{p q}+\mathbf{v}_{g} \cdot \nabla \Omega_{p q}, \\
s_{g}^{[B]}= & \frac{\partial^{2} e_{\mathrm{xc}}}{\partial \rho^{2}} \rho^{B}+\frac{\partial^{2} e_{\mathrm{xc}}}{\partial \rho \partial \zeta} \cdot 2\left(\nabla \rho \cdot \nabla \rho^{B}\right), \\
\mathbf{v}_{g}^{[B]}= & {\left[\frac{\partial^{2} e_{\mathrm{xc}}}{\partial \rho \partial \zeta} \rho^{B}+\frac{\partial^{2} e_{\mathrm{xc}}}{\partial \zeta^{2}} \cdot 2\left(\nabla \rho \cdot \nabla \rho^{B}\right)\right] \cdot 2 \nabla \rho } \\
& +\frac{\partial e_{\mathrm{xc}}}{\partial \zeta} \cdot 2 \nabla \rho^{B} .
\end{aligned}
$$

We will reach our desired expression for the quadratic response by performing a differentiation of Eq. (16) with respect to the external field. We collect the final result of this derivation in the form

$$
\left.\frac{\partial}{\partial \kappa_{a i}^{*}} \frac{d^{2} E_{\mathrm{xc}}}{d \varepsilon_{B} d \varepsilon_{C}}\right|_{\varepsilon=0}=-\int\left(f_{\mathrm{xc} ; a i}^{B C}+g_{\mathrm{xc} ; a i}^{[C] B}+g_{\mathrm{xc} ; a i}^{[B] C}+h_{\mathrm{xc} ; a i}^{[B C]}\right) d \tau,
$$

where we recognize the doubly one-index transformed exchange-correlation part of the Kohn-Sham matrix $f_{\mathrm{xc} ; p q}^{B C}$ as well as matrices $g_{\mathrm{xc} ; a i}^{[C] B}$ and $g_{\mathrm{xc} ; a i}^{[B] C}$ which are one-index transformed versions of Eq. (18) with respect to $B$ and $C$, respectively. The remainder is collected into

$$
\begin{aligned}
h_{\mathrm{xc} ; p q}^{[B C]} & =s_{h} \Omega_{p q}+\mathbf{v}_{h} \cdot \nabla \Omega_{p q}, \\
s_{h}= & \sum \mathcal{P}_{B, C}\left\{\frac{\partial^{3} e_{\mathrm{xc}}}{\partial \rho^{3}} \rho^{B} \rho^{C}+2 \frac{\partial^{3} e_{\mathrm{xc}}}{\partial \rho^{2} \partial \zeta} \rho^{B} \cdot 2\left(\nabla \rho \cdot \nabla \rho^{C}\right)\right. \\
& +\frac{\partial^{3} e_{\mathrm{xc}}}{\partial \rho \partial \zeta^{2}} \cdot 2\left(\nabla \rho \cdot \nabla \rho^{B}\right) \cdot 2\left(\nabla \rho \cdot \nabla \rho^{C}\right)+\frac{\partial^{2} e_{\mathrm{xc}}}{\partial \rho^{2}} \rho^{B C} \\
& \left.+\frac{\partial^{2} e_{\mathrm{xc}}}{\partial \rho \partial \zeta} \cdot 2\left[\left(\nabla \rho \cdot \nabla \rho^{B C}\right)+\left(\nabla \rho^{B} \cdot \nabla \rho^{C}\right)\right]\right\}, \\
\mathbf{v}_{h}= & \sum \mathcal{P}_{B, C}\left\{\left[\frac{\partial^{3} e_{\mathrm{xc}}}{\partial \zeta^{3}} \cdot 2\left(\nabla \rho \cdot \nabla \rho^{B}\right) \cdot 2\left(\nabla \rho \cdot \nabla \rho^{C}\right)\right.\right. \\
& +2 \frac{\partial^{3} e_{\mathrm{xc}}}{\partial \rho \partial \zeta^{2}} \rho^{B} \cdot 2\left(\nabla \rho \cdot \nabla \rho^{C}\right)+\frac{\partial^{3} e_{\mathrm{xc}}}{\partial \rho^{2} \partial \zeta} \rho^{B} \rho^{C}+\frac{\partial^{2} e_{\mathrm{xc}}}{\partial \rho \partial \zeta} \rho^{B C} \\
& +\frac{\partial^{2} e_{\mathrm{xc}}}{\partial \zeta^{2}} \cdot 2\left[\left(\nabla \rho \cdot \nabla \rho^{B C}\right)+\left(\nabla \rho^{B} \cdot \nabla \rho^{C}\right)\right] \cdot 2 \nabla \rho \\
& +\left[\frac{\partial^{2} e_{\mathrm{xc}}}{\partial \rho \partial \zeta} \rho^{C}+\frac{\partial^{2} e_{\mathrm{xc}}}{\partial \zeta^{2}} \cdot 2\left(\nabla \rho \cdot \nabla \rho^{C}\right)\right] \cdot 4 \nabla \rho^{B} \\
& \left.+\frac{\partial e_{\mathrm{xc}}}{\partial \zeta} \cdot 2 \nabla \rho^{B C}\right\},
\end{aligned}
$$

where the operator $\Sigma \mathcal{P}_{B, C}$ denotes the sum over permutations between $B$ and $C$. An implementation of Eq. (19) has been added to the DIRAC program ${ }^{35}$ and below, we will present an example calculation of the electric-dipole hyperpolarizability which corresponds to a quadratic response function [Eq. (4)] evaluated for electric-dipole operators. The implementation is general in the sense that it merely assumes the perturbations to be due to one-electron operators and our work is, therefore, in principle, applicable to a series of electromagnetic properties. However, the introduction of a magnetic perturbation breaks the time-reversal symmetry of the system and requires the consideration of spin polarization in the formalism. In fact, even the application of time-dependent electric fields will induce electronic currents and thereby magnetic fields, so also the evaluation of dynamic polarizabilities should take spin polarization into account. We will return to this topic in a future work.

\section{EXAMPLE CALCULATIONS}

\section{A. Computational details}

All calculations in the present work were performed for molecular structures that were optimized at the onecomponent Kohn-Sham DFT level of theory using the hybrid B3LYP exchange-correlation functional; ${ }^{36}$ for $\mathrm{H}, \mathrm{C}$, and $\mathrm{Br}$, 
the $6-31 G^{*}$ basis set was used, ${ }^{37,38}$ and for iodine, the Stuttgart ECP was used. ${ }^{39}$ Structure optimizations were performed in the $C_{2 v}$ point group with the GAUSSIAN program. ${ }^{40}$ The molecules are placed with the $z$ axis as principle axis and in the $y z$ plane with the heavy atoms along the negative $z$ direction.

The all-electron property calculations were performed with a locally modified version of the DIRAC program, ${ }^{35}$ and those where an ECP was used for $\mathrm{Br}$ or I were performed with a version of the DALTON program ${ }^{41}$ to which an implementation of the Coulomb attenuated B3LYP (Ref. 42) (CAM-B3LYP) has been added. ${ }^{43}$ The property calculations based on single determinant reference states (all-electron as well as ECP based) were performed with fully uncontracted basis sets that are based on the exponents from Sadlej's polarization basis set ${ }^{44}$ with further addition of polarization and diffuse functions. The basis sets were augmented using the formula

$$
\zeta_{N+j}=\left[\frac{\zeta_{N}}{\zeta_{N-1}}\right]^{j} \zeta_{N}, \quad j \in\left[1, N_{\mathrm{aug}}\right],
$$

where $N_{\text {aug }}$ is the number of augmentation functions added and $\zeta_{N}$ and $\zeta_{N-1}$ refer to the two most diffuse exponents in the original basis sets. The only exception to this rule is the $f$ shell of the iodine basis set, which was not augmented. To the basis set of bromine, we added four $f$ functions based on the four most diffuse $p$ exponents in the original basis set. The sizes of the singly augmented large component basis sets used in the property calculations were $[7 s 5 p],[11 s 7 p 5 d]$, $[16 s 13 p 10 d 4 f]$, and $[20 s 16 p 13 d 4 f]$ for $\mathrm{H}, \mathrm{C}, \mathrm{Br}$, and I, respectively, and the small-component basis functions were generated from those of the large component with the use of the restricted kinetic-balance condition. In all fourcomponent calculations, we have ignored the interactions between the small component densities, i.e., the $(S S \mid S S)$ integrals. This approximation has virtually no influence on the presented results, as demonstrated in Ref. 23, and will not be further discussed here. All DFT functionals were employed self-consistently and with their proper derivatives to the required orders in the perturbations.

For comparison, wave function correlated results were obtained at the coupled cluster level with inclusion of single and double electron excited configurations (CCSD). For these calculations, we adopted the contracted Sadlej basis $\mathrm{set}^{44}$ for hydrogen and carbon but augmented with the same diffuse functions as described above. For bromine and iodine, we employed the valence basis set of the Stuttgart ECPs (Ref. 39) but augmented and polarized using the functions from the Sadlej basis set and Eq. (21). The sizes of the heavy atom basis sets in the CCSD calculations were $[6 s 6 p 5 d 4 f] /[4 s 4 p 3 d 2 f]$ (bromine) and $[6 s 6 p 8 d 2 f] /[4 s 4 p 3 d 1 f]$ (iodine).

\section{B. Results and discussion}

With a molecular dipole moment aligned with the $z$ axis, the relevant experimental observable for second-harmonic generation is $\mu \bar{\beta},{ }^{45}$ where

$$
\bar{\beta}(-2 \omega ; \omega, \omega)=\frac{1}{5} \sum_{\alpha=x, y, z}\left(\beta_{z \alpha \alpha}+2 \beta_{\alpha z \alpha}\right) .
$$

In previous studies, we have shown that relativistic effects in heavy atom substituted $\pi$-conjugated systems are pronounced for the dipole moment as well as the first-order hyperpolarizability (but not for the linear polarizability); ${ }^{22,23}$ for bromobenzene, the effects are predominantly scalar relativistic in nature but for iodobenzene, scalar relativistic and spin-orbit effects are about equally important. ${ }^{23}$ Whereas changes due to relativity in the dipole moment can be attributed to changes in the chemical bond polarities, the effects on the hyperpolarizabilities are not as easily interpreted. The sum-over-states expression for $\beta$, which reads as

$$
\begin{aligned}
& \beta_{\alpha \beta \gamma}\left(-\omega_{\sigma} ; \omega_{1}, \omega_{2}\right) \\
& =\hbar^{-2} \sum \mathcal{P}_{-\sigma, 1,2} \sum_{k, l}, \frac{\left\langle 0\left|\hat{\mu}_{\alpha}\right| k\right\rangle\left\langle k\left|\overline{\hat{\mu}}_{\beta}\right| l\right\rangle\left\langle l\left|\hat{\mu}_{\gamma}\right| 0\right\rangle}{\left(\omega_{k}-\omega_{\sigma}\right)\left(\omega_{l}-\omega_{2}\right)},
\end{aligned}
$$

reveals an intricate dependence of the hyperpolarizability on interexcited state transition moments and excited-to-ground state dipole moment differences, in addition to a dependence on the linear absorption spectrum via the ground-to-excited state transition dipole moments and excitation energies. The permutation operator introduced in Eq. (23) permutes the pairs of dipole moment operators and optical frequencies $\left(\hat{\mu}_{\alpha},-\omega_{\sigma}\right),\left(\hat{\mu}_{\beta}, \omega_{1}\right)$, and $\left(\hat{\mu}_{\gamma}, \omega_{2}\right)$, and $\overline{\hat{\mu}}$ denotes the electricdipole fluctuation operator. One thing that becomes clear from the sum-over-states expression is the separation between scalar relativistic and spin-orbit effects, since the latter can be attributed to the coupling between states in the singlet and triplet manifolds. The nonrelativistic and relativistic linear absorption spectra presented in Ref. 23 show significant spin-forbidden absorption only for the iodobenzenes, and scalar relativistic and spin-orbit effects on $\beta$ are also of comparable magnitude in this case, whereas the spin-orbit effects are small on the same property for the bromobenzenes.

The same argumentation can be made for the linear polarizability. In fact, $\alpha$ depends only on the observables in the linear absorption spectrum, namely, transition energies and intensities. The absence of relativistic effects that are seen in Tables I and II for this property is, therefore, puzzling but has been previously noted also for the thiophene homologs which serve as important building blocks in optical materials. $^{22}$

In the present work, we focus at a formulation of the quadratic response function at the electron correlated fourcomponent level of theory. We give here a presentation of the response function which is quite different from the sumover-states expression in Eq. (23) but is rather seen as energy derivatives. Since the correlation energy depends on the electron density, its value per electron will basically be larger, the heavier the atom. What makes property calculations at the Hartree-Fock level at all reasonable for heavy atoms are the facts that the molecular property is a measure of the energy difference with respect to external fields and that the induced fluctuations in the core electron densities are very small-in the valence region, where density fluctuations are larger, the 
TABLE I. Optical properties for disubstituted bromobenzenes at the Hartree-Fock, Kohn-Sham, and post-HF levels of theory. Different exchange-correlation functionals are considered for the inclusion of electron correlation effects. All quantities are given in a.u.

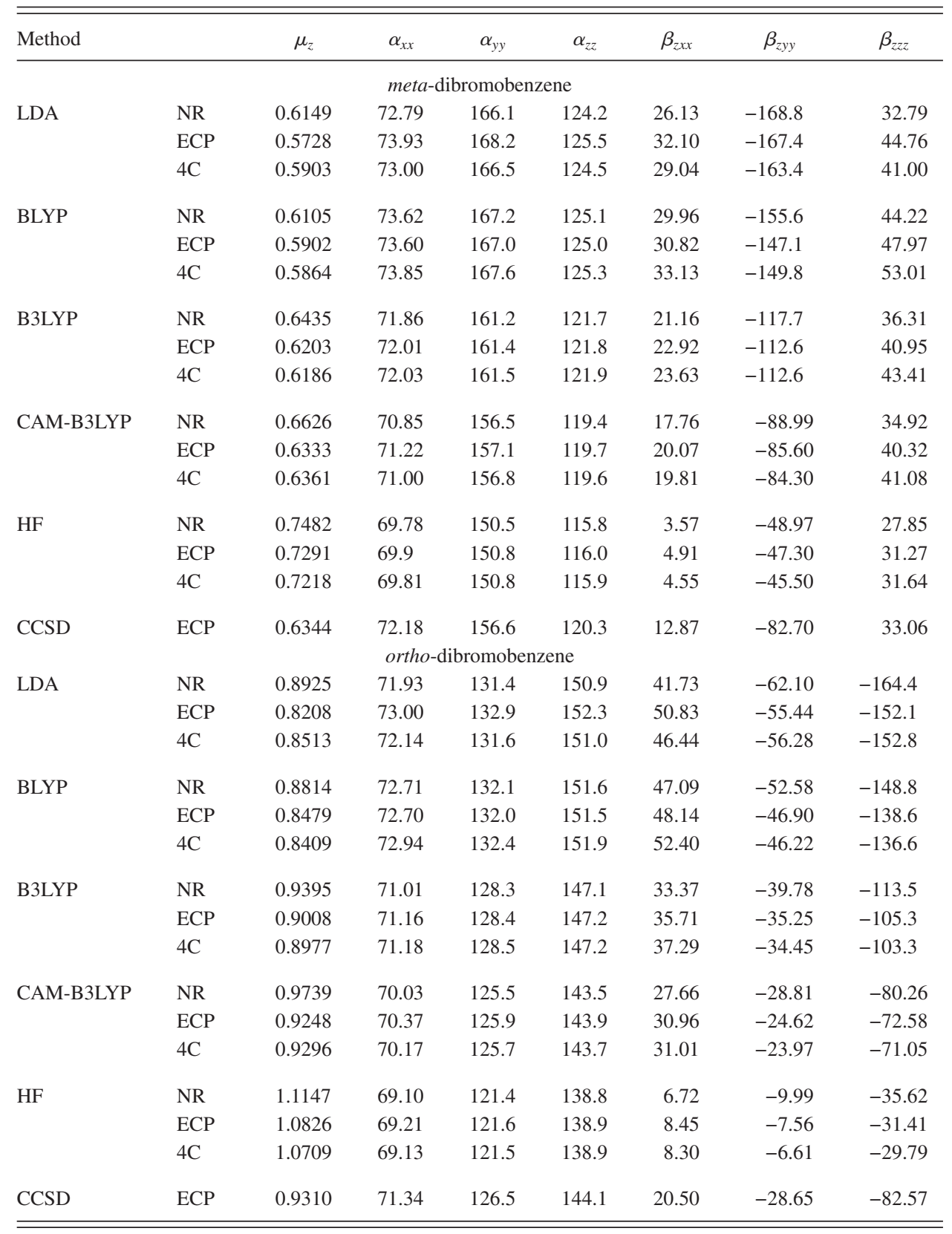

effects of electron correlation are smaller. As a rule of thumb for the polarizability of molecules containing first- and second-row elements, the isotropic value is typically underestimated by some $5 \%$ at the Hartree-Fock level of theory, whereas anisotropies suffer from larger errors. ${ }^{46}$ For the hyperpolarizabilities, on the other hand, the effects of electron correlation are known to be both large and unsystematic.

In Tables I and II, we present the optical properties of disubstituted bromobenzene and iodobenzene, respectively, in their meta and ortho conformations. We employ a series of four standard density functionals in the correlated fourcomponent calculations, namely, local-density approximation (LDA), BLYP, B3LYP, and CAM-B3LYP, and the ordering of functionals in the tables reflects the consensus of increasing accuracy as due to gradient and exact exchange correc- tions. An apparent consequence of the use of the more sophisticated density functionals is the improved quality of the orbital energies and, since the difference in orbital energies between virtual and occupied orbitals appears on the diagonal of the electronic Hessian, it will correspond to improved excitation energies in the response function approach as well. In Ref. 47, it was also well illustrated how the inclusion of exact exchange in the density functional affects linear response calculation of excitation energies. It is the exact exchange that provides the Coulomb attraction between the hole and the electron in these calculations, and the more spatially separated the hole and electron orbitals are, the greater the need for exact exchange in the functional is. The present systems are by no means extreme charge-transfer systems but, at the same time, it is clear that the halogen 
TABLE II. Optical properties for disubstituted iodobenzenes at the Hartree-Fock, Kohn-Sham, and post-hF levels of theory. Different exchange-correlation functionals are considered for the inclusion of electron correlation effects. All quantities are given in a.u.

\begin{tabular}{|c|c|c|c|c|c|c|c|c|}
\hline Method & & $\mu_{z}$ & $\alpha_{x x}$ & $\alpha_{y y}$ & $\alpha_{z z}$ & $\beta_{z x x}$ & $\beta_{z y y}$ & $\beta_{z z z}$ \\
\hline \multicolumn{9}{|c|}{ meta-di-iodobenzene } \\
\hline \multirow[t]{3}{*}{ LDA } & NR & 0.6337 & 94.24 & 214.0 & 149.1 & 85.17 & -175.1 & 140.6 \\
\hline & ECP & 0.5674 & 95.86 & 216.1 & 150.6 & 96.74 & -166.0 & 167.0 \\
\hline & $4 \mathrm{C}$ & 0.5661 & 94.61 & 215.1 & 149.5 & 96.78 & -151.2 & 171.4 \\
\hline \multirow[t]{3}{*}{ BLYP } & NR & 0.6240 & 95.86 & 216.0 & 150.5 & 95.56 & -150.4 & 166.4 \\
\hline & $\mathrm{ECP}$ & 0.5885 & 95.16 & 214.3 & 149.7 & 96.71 & -128.2 & 174.8 \\
\hline & $4 \mathrm{C}$ & 0.5575 & 96.32 & 217.4 & 151.1 & 108.7 & -125.6 & 200.3 \\
\hline \multirow[t]{3}{*}{ B3LYP } & NR & 0.6501 & 93.55 & 208.1 & 146.5 & 79.16 & -88.74 & 151.1 \\
\hline & $\mathrm{ECP}$ & 0.6076 & 93.30 & 207.4 & 146.2 & 82.61 & -73.72 & 162.0 \\
\hline & $4 \mathrm{C}$ & 0.5806 & 93.79 & 209.9 & 146.9 & 89.19 & -64.85 & 179.0 \\
\hline \multirow[t]{3}{*}{ CAM-B3LYP } & NR & 0.6618 & 91.88 & 201.2 & 143.4 & 68.39 & -37.66 & 141.1 \\
\hline & $\mathrm{ECP}$ & 0.6057 & 92.17 & 201.6 & 143.7 & 73.46 & -25.95 & 155.5 \\
\hline & $4 \mathrm{C}$ & 0.5867 & 91.99 & 202.2 & 143.7 & 76.96 & -15.23 & 165.7 \\
\hline \multirow[t]{3}{*}{$\mathrm{HF}$} & NR & 0.7537 & 91.23 & 196.1 & 140.5 & 56.17 & 12.08 & 140.0 \\
\hline & $\mathrm{ECP}$ & 0.7074 & 91.14 & 196.5 & 140.5 & 59.36 & 22.27 & 150.5 \\
\hline & $4 \mathrm{C}$ & 0.6793 & 90.91 & 197.2 & 140.5 & 61.26 & 31.21 & 157.2 \\
\hline CCSD & ECP & 0.5822 & 96.54 & 205.1 & 147.6 & 80.97 & -46.26 & 180.7 \\
\hline \multicolumn{9}{|c|}{ ortho-di-iodobenzene } \\
\hline \multirow[t]{3}{*}{ LDA } & NR & 0.8877 & 92.07 & 166.3 & 182.7 & 125.0 & -42.42 & -46.42 \\
\hline & $\mathrm{ECP}$ & 0.7777 & 93.59 & 167.9 & 184.0 & 142.3 & -26.39 & -16.02 \\
\hline & $4 \mathrm{C}$ & 0.7766 & 92.45 & 166.6 & 182.9 & 143.9 & -19.06 & -2.32 \\
\hline \multirow[t]{3}{*}{ BLYP } & NR & 0.8639 & 93.49 & 167.7 & 183.9 & 136.7 & -24.59 & -16.73 \\
\hline & $\mathrm{ECP}$ & 0.8071 & 92.87 & 166.1 & 182.7 & 138.2 & -8.59 & 5.99 \\
\hline & $4 \mathrm{C}$ & 0.7544 & 93.94 & 168.1 & 184.2 & 157.9 & 1.05 & 29.70 \\
\hline \multirow[t]{3}{*}{ B3LYP } & NR & 0.9103 & 91.31 & 162.4 & 178.6 & 113.4 & 1.21 & 25.32 \\
\hline & $\mathrm{ECP}$ & 0.8411 & 91.11 & 161.6 & 178.0 & 118.1 & 14.86 & 45.94 \\
\hline & $4 \mathrm{C}$ & 0.7962 & 91.57 & 162.7 & 178.8 & 129.7 & 25.04 & 67.06 \\
\hline \multirow[t]{3}{*}{ CAM-B3LYP } & NR & 0.9336 & 89.74 & 158.1 & 174.1 & 97.05 & 22.97 & 66.22 \\
\hline & ECP & 0.8415 & 90.03 & 158.2 & 174.2 & 104.6 & 36.07 & 86.91 \\
\hline & $4 \mathrm{C}$ & 0.8105 & 89.88 & 158.3 & 174.3 & 110.4 & 45.22 & 104.3 \\
\hline \multirow[t]{3}{*}{$\mathrm{HF}$} & NR & 1.0740 & 89.19 & 154.3 & 170.2 & 78.37 & 53.66 & 123.4 \\
\hline & $\mathrm{ECP}$ & 0.9973 & 89.13 & 154.2 & 170.1 & 82.94 & 65.23 & 139.0 \\
\hline & $4 \mathrm{C}$ & 0.9519 & 88.93 & 154.3 & 170.4 & 86.26 & 72.80 & 151.2 \\
\hline CCSD & ECP & 0.8023 & 94.14 & 162.7 & 177.7 & 117.3 & 23.97 & 89.22 \\
\hline
\end{tabular}

atoms will play the role of donors in excitations to the $\pi^{*}$ orbitals. We, therefore, anticipate that the use of the CAMB3LYP can have an impact on results, and we would argue that these results are the best ones at the four-component level of theory. We would also like to draw attention to the systematic decrease in $\alpha$ when comparing results obtained with the series of functionals BLYP, B3LYP, and CAMB3LYP. This trend is directly coupled to an increasing portion of exact exchange and thereby increased excitation energies of the system.

When measured against the four-component CAM$\mathrm{B} 3 \mathrm{LYP}$ results, the correlation contributions to $\beta_{z x x}, \beta_{z y y}$, and $\beta_{z z z}$ of meta-bromobenzene amount to $15.3,-38.8$, and 9.4 a.u., respectively, and for meta-iodobenzene, the corresponding values are $15.7,-46.4$, and 8.5 a.u. In both cases, there is thus a strong error cancellation for the Hartree-Fock values of the observable $\bar{\beta}$ since electron correlation lowers the value of the zyy component but increases the values of the other two components. This illustrates how unsystematic correlation effects can be for the first-order hyperpolarizability. On the other hand, we note that the correlation effects on the hyperpolarizabilities of the two meta systems are close in magnitude. That again indicates that it is the correlation energy in the valence region that matters for this property, and that this energy is almost the same in the two systems. If we make the same comparison for the two ortho systems, we see correlation contributions of $22.7,-17.4$, and -41.3 a.u. for the three nonzero $\beta$ components of bromobenzene and 24.1, $-27.6,-46.9$ a.u. for the three components of iodobenzene. 
We have argued that the ordering of DFT results in the tables reflects the quality. In order to get a more objective measure of the performance of the various functionals, we have also determined the optical properties using a nonrelativistic wave function correlated approach in conjunction with the Stuttgart relativistic ECPs. Due to the computational cost associated with the CCSD method, we are forced to employ a reduced basis set and, given the fact that the basis set requirements are stronger in wave function than in density functional approaches, we cannot use the CCSD results as benchmarks. Furthermore, the lack of inclusion of relativistic effects in the valence region will make the results based on ECPs error prone for the iodobenzenes. For the $\beta$-tensor elements of bromobenzenes, the largest discrepancy between $\mathrm{ECP}$ and four-component results at the CAM-B3LYP level is as small as 1.5 a.u. (or $2 \%$ ), whereas for the iodobenzenes, this error bar is 17.4 a.u. It is, therefore, reasonable to use the bromobenzene CCSD results for the evaluation of the various density functionals. For each individual $\beta$ component of the bromobenzenes, the best agreement with the CCSD results is obtained with use of the CAM-B3LYP functional but, at the same time, it is clear that discrepancies between the correlated results can be as large as 10 a.u. (see the $z z z$ component of ortho-bromobenzene).

The calculations of the hyperpolarizabilities of the halobenzenes amply demonstrate that electron correlation effects can be very large for this property. Of greater concern to the present work, however, is the fact that relativistic effects on the hyperpolarizability are substantial for the bromobenzenes and large for the iodobenzenes. The development of electron correlated propagator methods with proper inclusion of relativity is particular important since the two effects are not additive. Without exception for the $\beta$ tensor, the relativistic effects at the correlated level of theory exceed those at the uncorrelated level of theory, e.g., the relativistic effects for $\beta_{z x x}, \beta_{z y y}$, and $\beta_{z z z}$ at the CAM-B3LYP level amount to $13.3,22.2$, and 38.1 a.u., respectively, whereas at the Hartree-Fock level, the corresponding values are 7.9, 19.2 , and 27.8 a.u. The relativistic corrections are without exception positive, thereby increasing the value of $\bar{\beta}$.

\section{CONCLUSIONS}

A derivation and implementation of the quadratic response function at the four-component density functional level of theory has been presented. The adiabatic, Kramersrestricted Kohn-Sham approximation has been adopted with consideration made of gradient-corrected functionals. We exemplify the significance of this work with calculations of the optical properties of disubstituted halobenzenes and thereby illustrate internal heavy atom effects on the hyperpolarizabilities in $\pi$-conjugated systems. Our best results are obtained with the use of the Coulomb attenuated B3LYP functional, ${ }^{42}$ which here provides notably different hyperpolarizability values from B3LYP. It is shown that correlation as well as relativistic effects on $\beta$ are large for the systems under investigation. Relativity alone reduces the $\mu \bar{\beta}$-response signals by $62 \%$ and $75 \%$ for meta- and orthobromobenzene, respectively, and enhances the same response by $17 \%$ and $21 \%$ for meta- and ortho-iodobenzene, respectively (these values are based on the CAM-B3LYP results). The results in the present work also demonstrates the wellknown fact that correlation and relativistic effects are not additive and that our work is called for.

\section{ACKNOWLEDGMENTS}

We acknowledge the use of computational resources at the National Supercomputer Centre (NSC) in Linköping, Sweden.

${ }^{1}$ R. W. Boyd, Nonlinear Optics, 2nd ed. (Academic, New York, 2003).

${ }^{2}$ J. Olsen and P. Jørgensen, J. Chem. Phys. 82, 3235 (1985).

${ }^{3}$ H. Sekino and R. Bartlett, J. Chem. Phys. 85, 976 (1986).

${ }^{4}$ S. Karna and M. Dupuis, J. Comput. Chem. 12, 487 (1991).

${ }^{5}$ J. Rice, R. Amos, S. Colwell, N. Handy, and J. Sanz, J. Chem. Phys. 93, 8828 (1990)

${ }^{6}$ H. Hettema, H. Jensen, P. Jørgensen, and J. Olsen, J. Chem. Phys. 97, 1174 (1992)

${ }^{7}$ J. Rice and N. Handy, Int. J. Quantum Chem. 43, 91 (1992).

${ }^{8}$ C. Hättig, O. Christiansen, H. Koch, and P. Jørgensen, Chem. Phys. Lett. 269, 428 (1997).

${ }^{9}$ J. Gauss, O. Christiansen, and J. Stanton, Chem. Phys. Lett. 296, 117 (1998).

${ }^{10}$ P. Rozyczko and R. Bartlett, J. Chem. Phys. 107, 10823 (1997).

${ }^{11}$ J. Olsen, P. Jørgensen, T. Helgaker, and J. Oddershede, J. Phys. Chem. A 109, 11618 (2005).

${ }^{12}$ S. van Gisbergen, J. Snijders, and E. Baerends, J. Chem. Phys. 109, 10644 (1998); 111, 6652(E) (1999).

${ }^{13}$ P. Salek, O. Vahtras, T. Helgaker, and H. Ågren, J. Chem. Phys. 117, 9630 (2002).

${ }^{14}$ P. Norman, D. M. Bishop, H. J. Aa. Jensen, and J. Oddershede, J. Chem. Phys. 123, 194103 (2005).

${ }^{15}$ D. Roberto, R. Ugo, E. Tessore, F. Lucenti, S. Quici, S. Vezza, P. Fantucci, I. Invernizzi, S. Bruni, I. Ledoux-Rak, and J. Zyss, Organometallics 21, 161 (2002).

${ }^{16}$ O. Vahtras, H. Ågren, P. Jørgensen, H. J. Aa. Jensen, T. Helgaker, and J. Olsen, J. Chem. Phys. 97, 9178 (1992).

${ }^{17}$ I. Tunell, Z. Rinkevicius, O. Vahtras, P. Salek, T. Helgaker, and H. Ågren, J. Chem. Phys. 119, 11024 (2003).

${ }^{18}$ M. Engström, O. Vahtras, and H. Ågren, Chem. Phys. Lett. 328, 483 (2000).

${ }^{19}$ P. Manninen, K. Ruud, P. Lantto, and J. Vaara, J. Chem. Phys. 122, 114107 (2005).

${ }^{20}$ L. R. Kahn, P. Baybutt, and D. G. Truhlar, J. Chem. Phys. 65, 3826 (1976).

${ }^{21}$ P. Norman, B. Schimmelpfennig, K. Ruud, H. J. Aa. Jensen, and H. Ågren, J. Chem. Phys. 116, 6914 (2002).

${ }^{22}$ B. Jansik, B. Schimmelpfennig, P. Norman, H. Ågren, and K. Ohta, J. Mol. Struct.: THEOCHEM 633, 237 (2003).

${ }^{23}$ J. Henriksson, U. Ekström, and P. Norman, J. Chem. Phys. 124, 214311 (2006).

${ }^{24}$ J. Henriksson, P. Norman, and H. J. Aa. Jensen, J. Chem. Phys. 122, 114106 (2005).

${ }^{25}$ S. Raptis, M. Papadopoulos, and A. Sadlej, J. Chem. Phys. 111, 7904 (1999).

${ }^{26}$ M. Douglas and N. Kroll, Ann. Phys. (N.Y.) 82, 89 (1974).

${ }^{27}$ G. Jansen and B. Hess, Phys. Rev. A 39, 6016 (1989).

${ }^{28}$ E. Tellgren, J. Henriksson, and P. Norman, J. Chem. Phys. 126, 064313 (2007).

${ }^{29}$ P. Norman and H. J. Aa. Jensen, J. Chem. Phys. 121, 6145 (2004).

${ }^{30}$ P. Salek, T. Helgaker, and T. Saue, Chem. Phys. 311, 187 (2005).

${ }^{31}$ T. Saue and T. Helgaker, J. Comput. Chem. 23, 814 (2002).

${ }^{32}$ T. Saue, K. Fægri, T. Helgaker, and O. Gropen, Mol. Phys. 91, 937 (1997).

${ }^{33}$ O. Christiansen, P. Jørgensen, and C. Hättig, Int. J. Quantum Chem. 68, 1 (1998).

${ }^{34}$ N. T. Maitra and K. Burke, Chem. Phys. Lett. 441, 167 (2007).

${ }^{35}$ H. J. Aa. Jensen, T. Saue, L. Visscher, V. Bakken, E. Eliav, T. Enevoldsen, T. Fleig, O. Fossgaard, T. Helgaker, J. Laerdahl et al., DIRAC, a relativistic ab initio electronic structure program, release as DIRAC4.0, 
2004.

${ }^{36}$ A. D. Becke, J. Chem. Phys. 98, 5648 (1993).

${ }^{37}$ W. J. Hehre, R. Ditchfield, and J. A. Pople, J. Chem. Phys. 56, 2257 (1972).

${ }^{38}$ V. A. Rassolov, J. A. Pople, M. A. Ratner, and T. L. Windus, J. Chem. Phys. 109, 1223 (1998).

${ }^{39}$ M. Kaupp, P. Schleyer, H. Stoll, and H. Preuss, J. Am. Chem. Soc. 113, 6012 (1991).

${ }^{40}$ M. J. Frisch, G. W. Trucks, H. B. Schlegel et al., GAUSSIAN 03, revision B05, Gaussian, Inc., Pittsburgh, PA, 2003.
${ }^{41}$ DALTON, a molecular electronic structure program, release 2.0, 2005 (see http://www.kjemi.uio.no/software/dalton/dalton.html).

${ }^{42}$ T. Yanai, D. P. Tew, and N. C. Handy, Chem. Phys. Lett. 393, 51 (2004).

${ }^{43}$ M. J. G. Peach, T. Helgaker, P. Salek, T. W. Keal, O. B. Lutnæs, D. J. Tozer, and N. C. Handy, Phys. Chem. Chem. Phys. 8, 558 (2006).

${ }^{44}$ A. J. Sadlej, Collect. Czech. Chem. Commun. 53, 1995 (1988).

${ }^{45}$ D. Shelton and J. Rice, Chem. Rev. (Washington, D.C.) 94, 3 (1994).

${ }^{46}$ S. A. C. McDowell, R. D. Amos, and N. C. Handy, Chem. Phys. Lett. 235, 1 (1995).

${ }^{47}$ A. Dreuw and M. Head-Gordon, J. Am. Chem. Soc. 126, 4007 (2004). 